\title{
Macroelement content in plants after amendment application to cobalt-contaminated soil
}

\author{
Milena Kosiorek $^{1} \cdot$ Mirosław Wyszkowski ${ }^{1}$ if \\ Received: 3 September 2020 / Accepted: 16 February 2021 / Published online: 26 February 2021 \\ (C) The Author(s) 2021
}

\begin{abstract}
Aim The study was undertaken to determine the effect of amendments used in remediation of cobalt-contaminated soil on the macroelement content of all organs of spring barley (the main crop) and white mustard (the after-crop).

Methods In the experiment, six blocks were selected: without amendments; with manure (bovine, granulated); clay; charcoal; zeolite; and with calcium oxide (50\%). In each of the blocks, increasing doses of cobalt were applied: 0, 20, 40, 80, 160, and $320 \mathrm{mg} \mathrm{kg}^{-1}$ of soil.

Results Cobalt-contaminated soil and amendments application had a significant effect on macroelement content of all organs of both plants.

Conclusions In blocks without amendments, $80 \mathrm{mg} \mathrm{Co} \mathrm{kg}^{-1}$ contributed the most increase in nitrogen, sodium, and calcium contents of both organs of spring barley. Cobalt-contaminated soil increased content of all macroelements in white mustard organs. Application of amendments to soil had strong impact on phosphorus, sodium, and calcium contents of organs of both plant species, compared to the control block (without amendments). Among the amendments, manure had the greatest effect on the content of macroelements in plants. Manure increased phosphorus, potassium, and sodium contents of all organs of both plants. The optimal content of macroelements is extremely important for the growth and development of plants, especially on soils contaminated with trace elements, including cobalt.
\end{abstract}

Keywords Cobalt $\cdot$ Amendments $\cdot$ Spring barley $\cdot$ White mustard $\cdot$ Macroelements

\section{Introduction}

The dry matter of plants contains both essential and unnecessary minerals as well as minerals with yet-unknown modes of operation (Stanisławska-Glubiak and Korzeniowska 2007). In terms of chemical properties, plant species, biochemical functions, and the volume of demand, minerals are usually divided into two or more categories (Loomis and Shull 1937). The essential minerals are referred to as macroelements (which are found in large quantities in plant tissues) and include nitrogen, phosphorus, potassium, magnesium, calcium, and sulphur. On the other hand, a significant role in the composition of plants,

Responsible editor: Girish Choppala

Mirosław Wyszkowski

miroslaw.wyszkowski@uwm.edu.pl

1 Department of Environmental Chemistry, University of Warmia and Mazury in Olsztyn, Łódzki 4 Sq., 10-727 Olsztyn, Poland the structure and activation of a variety of enzymes (copper, iron, molybdenum, manganese, zinc, and nickel), osmoregulation, metabolic regulation (chlorine, copper, manganese, iron, zinc), electron carriers, and increasing the resistance to both biotic and abiotic stress, is played by microelements (Sharma 2006). Ensuring the proper microelement content of a plant allows the effective use of essential macroelements during a biomass increase to be made (Stanisławska-Glubiak and Korzeniowska 2007; Brodowska and Kaczor 2009). A variety of factors have a strong effect on microelement uptake by plants in quantities necessary to ensure their proper growth and development. Most often, they include a number of soil properties, moisture content, atmospheric conditions, and their quantity in the soil (Ronan 2007; Gąsiorowska et al. 2011).

One of the microelements which may be found in certain soils in elevated quantities that poses a potential hazard to the development of plants is cobalt (Chatterjee and Chatterjee 2000). In addition to its naturally high content, which may be found in soils, the great accumulation thereof is most 
influenced by activities involving mining, processing, and the use of this metal in a variety of technological solutions (ATSDR 2004). The vast majority of cobalt is obtained as a by-product in the mining of copper and nickel (van den Brink et al. 2020). Therefore, places such as areas adjacent to roads or industrial and power distribution plants are most exposed to contamination with cobalt (ATSDR 2004). With great cobalt accumulation in the soil, the $\mathrm{pH}$ value can decrease, which results in more intense uptake by plants (Gál et al. 2008; Kosiorek and Wyszkowski 2016). It also has an adverse effect on the microbiological and biochemical activity of soils (Zaborowska et al. 2016). The occurrence in a plant of excessive quantities of microelements, which include cobalt as well, may have an effect on the occurrence of interactions between the other accumulated elements (Ronan 2007). The toxicity of cobalt to plants is related to inhibition of photosynthesis and iron deficiency as well as oxidative stress (Morrissey et al. 2009). There are plants which exhibit no toxic symptoms when significant quantities of accumulated cobalt are present, for example Alyssum murale or Bulbostylis pseudoperennis (Collins and Kinsela 2011; Saad et al. 2012), but cereals, for example, belong to the group of plants sensitive to contamination with this element (Kabata-Pendias and Pendias 2001). Cobalt accumulation and cobalt tolerance mechanisms are still poorly understood (Lange et al. 2017). One of the ways to reduce the content of this metal in the soil and to restore balance in the environment through, inter alia, increasing the availability of other elements with great potential significance, is phytoremediation treatments (Pszczółkowski et al. 2012). These treatments often involve the use of energy plants which are not used to produce food (Grzesik and Romanowska-Duda 2009) but also, inter alia, various varieties of mustard which belong to the group of plants known as hyperaccumulators (Baker et al. 1994). Phytoremediation is increasingly used to remove trace elements from contaminated soils (Awa and Hadibarata 2020; Saleh et al. 2019). A significant role is also attributed to operations associated with the use of a variety of soil-applied amendments improving the physico-chemical properties of soils, which consequently have an effect on increases in metals availability for plants (Kwiatkowska and Maciejewska 2008).

Therefore, studies were conducted to determine the impact of amendments used in the remediation of cobaltcontaminated soil on the macroelement content of all organs of spring barley and white mustard.

\section{Materials and methods}

\subsection{Methodological approach}

The vegetation pot experiment was established in vegetation hall belonging to University of Warmia and Mazury (Poland).
The research was carried out in polyethylene pots $(9 \mathrm{~kg}$ of soil) in Olsztyn (north-eastern Poland). The soil used in experiment was characterised by granulometric composition of loamy sand. Detailed soil and amendments characteristics were included in the previously published paper (Kosiorek and Wyszkowski 2020). In the experiment, six blocks were selected: without amendments; with manure (bovine, granulated); with clay; with charcoal; with zeolite (cliniptilolite); and with calcium oxide (50\%). Most of amendments (manure, clay, charcoal, zeolite) were applied at $2 \%$ of the amount of soil. The exception was calcium oxide, which was added to the soil in an amount equivalent to 1 hydrolytic acidity $(1.30 \mathrm{~g}$ $\mathrm{CaO} \mathrm{kg}^{-1}$ ). In each blocks with above-mentioned amendments, increasing doses of cobalt $\left(\mathrm{CoCl}_{2}\right)$ were applied: 0 , 20, 40, 80,160, and $320 \mathrm{mg} \mathrm{kg}^{-1}$ of soil. Moreover, the following were also introduced to each pot in one dose: $100 \mathrm{mg}$ of nitrogen, $35 \mathrm{mg}$ of phosphorus, $100 \mathrm{mg}$ of potassium, $50 \mathrm{mg}$ of magnesium, $0.33 \mathrm{mg}$ of boron, $5 \mathrm{mg}$ of manganese, and $5 \mathrm{mg}$ of molybdenum per $\mathrm{kg}$ of soil. Nitrogen was used as $\left(\mathrm{NH}_{4} \mathrm{NO}_{3}\right)$, phosphorus as $\mathrm{KH}_{2} \mathrm{PO}_{4}$, potassium as $\mathrm{KH}_{2} \mathrm{PO}_{4}+$ $\mathrm{KCl}$, magnesium as $\mathrm{MgSO}_{4} \cdot 7 \mathrm{H}_{2} \mathrm{O}$, boron as $\mathrm{H}_{3} \mathrm{BO}_{3}$, manganese as $\mathrm{MnCl}_{2} \cdot 4 \mathrm{H}_{2} \mathrm{O}$, and molybdenum as $\left(\mathrm{NH}_{4}\right)_{6} \mathrm{Mo}_{7} \mathrm{O}_{24}$. $4 \mathrm{H}_{2} \mathrm{O}$. The above-mentioned soil amendments, cobalt, and elements were applied while setting up the experiment (in three replications). The amendments were selected based on their positive effect on physico-chemical properties of soil, while the selection of cobalt doses was based on the cobalt acceptable levels in soil (Regulation 2016). Cobalt doses are multiples of the acceptable cobalt level $\left(20 \mathrm{mg} \mathrm{kg}^{-1}\right)$ in agricultural soils in Poland. The spring barley (Hordeum vulgare L.) and white mustard (Sinapis alba L.) were test plants. The spring barley (main crop) var. Mercada was sowing in density of 15 plants per pot. The spring barley was harvested in the heading stage, after 52 days from sowing. Next, the white mustard var. Bamberka (after-crop) was sowing to the same soil in density of 9 plants per pot. After 36 days, in the flowering stage, all organs of white mustard were harvested. The selection of spring barley for the study was based on its high percentage in the cereal cropping pattern in Poland; on the other hand, white mustard was selected due to the recommendations for sowing this plant as an aftercrop (following cereal cultivation) and its hyperaccumulatory properties towards metals found in the soil. The content of cobalt in plants was previously published in paper Kosiorek and Wyszkowski (2020).

\subsection{Methods of laboratory and statistical analyses}

The harvested shoots and roots of both test plants were dried at $60^{\circ} \mathrm{C}$ and then ground in an electric grinder. The plant material was subjected to a "wet" mineralisation procedure in concentrated (95\%) $\mathrm{H}_{2} \mathrm{SO}_{4}$. The following contents were then determined: total nitrogen — by Kjeldahl method (Bremner 1965), 
phosphorus — by colorimetric method (Ostrowska et al. 1991), other macroelements $(\mathrm{K}, \mathrm{Ca}, \mathrm{Mg}, \mathrm{Na})$ - by AAS (atomic absorption spectroscopy) method (Ostrowska et al. 1991). The Statistica 13 package (Dell Inc. 2016) with ANOVA variance analysis, correlation coefficients $(r)$, and the PCA analysis were used for the statistical analysis of research results.

\section{Results}

The cobalt introduced to the soil and all amendments in form of manure, clay, charcoal, zeolite, and calcium oxide had an effect on macroelement contents of all organs of test plants.

\subsection{Nitrogen}

In the objects without amendments, the 3rd cobalt dose ( $80 \mathrm{mg} \mathrm{Co} \mathrm{kg}^{-1}$ of soil) had maximum effect on an increase in nitrogen content of spring barley shoots $(17 \%, r=0.93)$ and roots $(28 \%, r=0.86)$ and in white mustard shoots $(53 \%$, $r=0.92)$; in turn, 1 st cobalt dose (20 mg Co kg ${ }^{-1}$ of soil) had the uttermost impact on its accumulation in white mustard roots $(50 \%, r=0.45)$ (Table 1). The introduction of all amendments to soil had little effect on nitrogen content of spring barley shoots. Clay had the strongest impact on an increase in the content of nitrogen in roots of this plant (9\%), compared to the control block (no amendments). The applied amendments had no significant impact on nitrogen content of white mustard, while charcoal contributed to the greatest reduction, both in shoots $(11 \%)$ and in roots $(23 \%)$. Zeolite and calcium oxide also reduced the nitrogen content of white mustard.

\subsection{Phosphorus}

In the blocks without amendments, contamination of the soil with 3 rd cobalt dose $\left(80 \mathrm{mg} \mathrm{Co} \mathrm{kg}{ }^{-1}\right)$ contributed to maximum reduction in phosphorus content $(22 \%, r=-0.94)$ in spring barley shoots, and an increase in this macroelement content in roots of this plant $(13 \%, r=0.98)$ and in white mustard shoots $(34 \%, r=0.94)$ (Table 2). After the application of 2nd cobalt dose (40 mg Co kg-1 of soil), a 9\% ( $r=0.55)$ increase in phosphorus content of white mustard roots was noted. The highest increase in the phosphorus content of spring barley shoots was demonstrated after the introduction of zeolite to soil (46\%), and in roots - after introduction of manure (56\%), compared to the control block (without amendments). Manure also had an effect on an increase in its content in aftercrop shoots (12\%) and roots (25\%). In other blocks, a decrease in phosphorus content of after-crop was observed, especially in objects with $\mathrm{CaO}$. In calcium oxide blocks, a decrease in its content of white mustard shoots $(16 \%)$ and roots $(15 \%)$ was noted. Charcoal exhibited a similar action in shoots and clay in roots of white mustard.

\subsection{Potassium}

In the control blocks, the application of $80 \mathrm{mg} \mathrm{Co} \mathrm{kg}^{-1}$ of soil increased potassium content of shoots of spring barley (7\%, $r=0.96)$ and white mustard $(17 \%, r=0.93)$; in turn, introduction of $40 \mathrm{mg} \mathrm{Co} \mathrm{kg}^{-1}$ of soil decreased phosphorus content of spring barley roots $(8 \%, r=-0.76)$, and its increased in white mustard roots $(28 \%, r=0.95)$ (Table 3$)$. In the objects with charcoal, a slight potassium increase was noted in main crop shoots and a greater increase was noted in this plant roots $(11 \%)$ and in after-crop shoots $(6 \%)$, compared to the control block (no amendments). Addition of manure to soil had the greatest effect on an increase in potassium content of white mustard roots (10\%). Among applied amendments, only zeolite had a strongest decreasing effect on content of this macroelement, both in shoots $(7 \%)$ and roots (7\%) of spring barley and in shoots $(8 \%)$ and roots $(20 \%)$ of white mustard.

\subsection{Sodium}

In the objects without amendments, a 3rd cobalt dose $(80 \mathrm{mg}$ Co $\mathrm{kg}^{-1}$ of soil) had the greatest effect on the increase in sodium content of spring barley shoots $(28 \%, r=1.00)$ and roots $(35 \%, r=0.98)$, while a 2 nd cobalt dose ( $40 \mathrm{mg}$ Co $\mathrm{kg}^{-1}$ ) reduced by half also contributed to the increase in this macroelement content in white mustard shoots $(20 \%, r=0.73)$ and roots $(16 \%, r=0.94)$ (Table 4). The soil application of zeolite resulted in an increase in sodium content of spring barley shoots (on average, by 107\%) and roots (by 66\%) and its content of white mustard shoots (by 71\%) and roots (by 45\%), compared to the control block (without amendments). Charcoal exerted an opposite effect in the objects with this addition and the sodium content of white mustard shoots decreased by $11 \%$ and in its roots by $13 \%$. Reverse relationships were observed in blocks with manure in all parts of plants and in objects with charcoal and calcium oxide in shoots of spring barley.

\subsection{Calcium}

In the blocks without amendments, a 3rd cobalt dose ( $80 \mathrm{Co}$ $\mathrm{kg}^{-1}$ of soil) had a significant effect on an increase in calcium content of main crop shoots $(26 \%, r=0.67)$ and roots $(133 \%$, $r=0.96)$ as well as its content in after-crop shoots $(49 \%$, $r=0.99$ ) (Table 5). When the soil was contaminated with a 2nd cobalt dose (40 mg Co kg-1 of soil), a highly significant increase $(112 \%, r=0.94)$ in calcium content in white mustard roots was noted. The introduction of calcium oxide to soil resulted in an increase in content of calcium in tested plant parts. In spring barley shoots, it was $46 \%$, in its roots $59 \%$, and in white mustard roots of $37 \%$, compared to the control block (no amendments). It was low in 
Table 1 Nitrogen content in plants

\begin{tabular}{|c|c|c|c|c|c|c|c|}
\hline \multirow{2}{*}{$\begin{array}{l}\text { Cobalt dose (mg kg} \\
\text { of soil) }\end{array}$} & \multicolumn{7}{|c|}{ Kind of amendment } \\
\hline & $\begin{array}{l}\text { Without } \\
\text { amendments }\end{array}$ & Manure & Clay & Charcoal & Zeolite & $\begin{array}{l}\text { Calcium } \\
\text { oxide }\end{array}$ & Average \\
\hline \multicolumn{8}{|l|}{ Spring barley—shoots } \\
\hline 0 & 27.41 & 27.83 & 26.39 & 27.83 & 28.95 & 25.73 & 27.35 \\
\hline 20 & 27.76 & 28.12 & 26.16 & 28.49 & 28.25 & 28.07 & 27.81 \\
\hline 40 & 28.00 & 27.98 & 28.82 & 28.40 & 28.58 & 28.74 & 28.42 \\
\hline 80 & 32.01 & 29.12 & 30.15 & 30.40 & 29.77 & 28.46 & 29.98 \\
\hline 160 & n.a. & 29.79 & 37.94 & 35.18 & 33.62 & 29.14 & 33.13 \\
\hline 320 & n.a. & 35.96 & n.a. & n.a. & n.a. & 34.56 & 35.26 \\
\hline Average & 28.79 & 29.80 & 29.89 & 30.06 & 29.83 & 29.11 & 30.33 \\
\hline$r$ & 0.93 & 0.97 & 0.98 & 0.98 & 0.94 & 0.94 & 0.97 \\
\hline $\operatorname{LSD}_{(0.01)}$ & \multicolumn{7}{|c|}{ Cobalt dose -0.90 , kind of amendment -0.90 , interaction -2.20} \\
\hline \multicolumn{8}{|l|}{ Spring barley—roots } \\
\hline 0 & 20.67 & 23.57 & 23.21 & 21.86 & 22.68 & 21.84 & 22.30 \\
\hline 20 & 20.60 & 19.92 & 23.63 & 22.72 & 19.11 & 20.37 & 21.06 \\
\hline 40 & 20.37 & 19.50 & 22.70 & 20.53 & 23.98 & 21.19 & 21.38 \\
\hline 80 & 26.53 & 22.45 & 26.83 & 23.73 & 23.26 & 21.81 & 24.10 \\
\hline 160 & n.a. & 23.94 & n.a. & n.a. & n.a. & 24.94 & 24.44 \\
\hline 320 & n.a. & 26.95 & n.a. & n.a. & n.a. & n.a. & 26.95 \\
\hline Average & 22.04 & 22.72 & 24.09 & 22.21 & 22.26 & 22.03 & 23.37 \\
\hline$r$ & 0.86 & 0.80 & 0.81 & 0.47 & 0.39 & 0.87 & 0.93 \\
\hline $\operatorname{LSD}_{(0.01)}$ & \multicolumn{7}{|c|}{ Cobalt dose -0.45 , kind of amendment -0.45 , interaction -1.10} \\
\hline \multicolumn{8}{|l|}{ White mustard—shoots } \\
\hline 0 & 32.80 & 37.92 & 33.64 & 33.32 & 37.99 & 35.32 & 35.16 \\
\hline 20 & 33.95 & 38.80 & 39.10 & 30.08 & 33.34 & 35.88 & 35.19 \\
\hline 40 & 47.02 & 41.79 & 44.84 & 34.37 & 37.70 & 36.63 & 40.39 \\
\hline 80 & 50.28 & 44.70 & 47.97 & 47.39 & 40.44 & 38.03 & 44.80 \\
\hline 160 & n.a. & 51.96 & n.a. & n.a. & n.a. & 46.17 & 49.06 \\
\hline 320 & n.a. & n.a. & n.a. & n.a. & n.a. & n.a. & n.a. \\
\hline Average & 41.01 & 43.03 & 41.38 & 36.29 & 37.37 & 38.40 & 40.92 \\
\hline$r$ & 0.92 & 1.00 & 0.95 & 0.88 & 0.59 & 0.97 & 0.96 \\
\hline $\operatorname{LSD}_{(0.01)}$ & \multicolumn{7}{|c|}{ Cobalt dose -1.72 , kind of amendment -1.72 , interaction -4.22} \\
\hline \multicolumn{8}{|l|}{ White mustard—roots } \\
\hline 0 & 14.02 & 16.61 & 12.69 & 12.85 & 14.63 & 13.77 & 14.09 \\
\hline 20 & 21.02 & 15.16 & 14.14 & 12.95 & 13.74 & 12.39 & 14.90 \\
\hline 40 & 17.20 & 18.25 & 18.22 & 14.37 & 15.47 & 14.93 & 16.41 \\
\hline 80 & n.a. & 18.81 & n.a. & n.a. & n.a. & 14.82 & 16.82 \\
\hline 160 & n.a. & n.a. & n.a. & n.a. & n.a. & n.a. & n.a. \\
\hline 320 & n.a. & n.a. & n.a. & n.a. & n.a. & n.a. & n.a. \\
\hline Average & 17.41 & 17.21 & 15.02 & 13.39 & 14.61 & 13.98 & 15.55 \\
\hline$r$ & 0.45 & 0.76 & 0.96 & 0.90 & 0.48 & 0.61 & 0.93 \\
\hline $\operatorname{LSD}_{(0.01)}$ & \multicolumn{7}{|c|}{ Cobalt dose -0.46 , kind of amendment -0.46 , interaction -1.12} \\
\hline
\end{tabular}

$r$, correlation coefficient; $n . a$., not analysed shoots of white mustard. Clay, manure, and charcoal had a positive effect on calcium content of spring barley roots. Zeolite had a positive effect on its content in white mustard roots. After the application of manure, calcium content of spring barley shoots decreased by $48 \%$, while in white mustard shoots by $17 \%$. Charcoal, zeolite, and manure contributed to decrease in the calcium content in white mustard shoots. 
Table 2 Phosphorus content in plants

\begin{tabular}{|c|c|c|c|c|c|c|c|}
\hline \multirow{2}{*}{$\begin{array}{l}\text { Cobalt dose ( } \mathrm{mg} \mathrm{kg}^{-1} \\
\text { of soil) }\end{array}$} & \multicolumn{7}{|c|}{ Kind of amendment } \\
\hline & $\begin{array}{l}\text { Without } \\
\text { amendments }\end{array}$ & Manure & Clay & Charcoal & Zeolite & $\begin{array}{l}\text { Calcium } \\
\text { oxide }\end{array}$ & Average \\
\hline \multicolumn{8}{|l|}{ Spring barley—shoots } \\
\hline 0 & 1.25 & 1.53 & 1.19 & 1.38 & 1.69 & 1.42 & 1.41 \\
\hline 20 & 1.11 & 1.62 & 1.17 & 1.53 & 1.81 & 1.52 & 1.46 \\
\hline 40 & 1.14 & 1.90 & 1.01 & 1.16 & 1.67 & 1.41 & 1.38 \\
\hline 80 & 0.97 & 1.58 & 1.30 & 1.28 & 1.56 & 1.59 & 1.38 \\
\hline 160 & n.a. & 1.46 & 1.37 & 1.37 & 1.41 & 1.16 & 1.35 \\
\hline 320 & n.a. & 1.41 & n.a. & n.a. & n.a. & 1.28 & 1.35 \\
\hline Average & 1.12 & 1.58 & 1.21 & 1.35 & 1.63 & 1.40 & 1.39 \\
\hline$r$ & -0.94 & -0.59 & 0.70 & -0.13 & -0.92 & -0.59 & -0.74 \\
\hline $\operatorname{LSD}_{(0.01)}$ & \multicolumn{7}{|c|}{ Cobalt dose -0.08 , kind of amendment $-0,08$, interaction -0.19} \\
\hline \multicolumn{8}{|l|}{ Spring barley—roots } \\
\hline 0 & 1.10 & 1.21 & 1.53 & 1.24 & 1.20 & 1.20 & 1.25 \\
\hline 20 & 1.12 & 1.82 & 1.57 & 1.19 & 1.07 & 1.20 & 1.33 \\
\hline 40 & 1.19 & 1.88 & 1.71 & 1.10 & 1.37 & 1.14 & 1.40 \\
\hline 80 & 1.24 & 2.00 & 1.39 & 1.18 & 1.44 & 1.30 & 1.43 \\
\hline 160 & n.a. & 1.93 & n.a. & n.a. & n.a. & 1.28 & 1.60 \\
\hline 320 & n.a. & 2.00 & n.a. & n.a. & n.a. & n.a. & 2.00 \\
\hline Average & 1.16 & 1.81 & 1.55 & 1.18 & 1.27 & 1.22 & 1.50 \\
\hline$r$ & 0.98 & 0.55 & -0.45 & -0.41 & 0.79 & 0.68 & 1.00 \\
\hline $\operatorname{LSD}_{(0.01)}$ & \multicolumn{7}{|c|}{ Cobalt dose -0.04 , kind of amendment -0.04 , interaction -0.09} \\
\hline \multicolumn{8}{|l|}{ White mustard—shoots } \\
\hline 0 & 1.55 & 1.79 & 1.49 & 1.60 & 1.69 & 1.55 & 1.61 \\
\hline 20 & 1.82 & 1.88 & 1.73 & 1.29 & 1.70 & 1.55 & 1.66 \\
\hline 40 & 1.78 & 1.92 & 1.73 & 1.59 & 1.83 & 1.47 & 1.72 \\
\hline 80 & 2.07 & 1.87 & 2.01 & 1.84 & 1.94 & 1.45 & 1.86 \\
\hline 160 & n.a. & 2.63 & n.a. & n.a. & n.a. & 1.58 & 2.11 \\
\hline 320 & n.a. & n.a. & n.a. & n.a. & n.a. & n.a. & n.a. \\
\hline Average & 1.81 & 2.02 & 1.74 & 1.58 & 1.79 & 1.52 & 1.79 \\
\hline$r$ & 0.94 & 0.91 & 0.97 & 0.67 & 0.97 & 0.24 & 1.00 \\
\hline $\operatorname{LSD}_{(0.01)}$ & \multicolumn{7}{|c|}{ Cobalt dose -0.08, kind of amendment -0.08 , interaction -0.19} \\
\hline \multicolumn{8}{|l|}{ White mustard—roots } \\
\hline 0 & 1.03 & 1.19 & 0.83 & 1.03 & 1.13 & 0.96 & 1.03 \\
\hline 20 & 0.96 & 1.17 & 0.94 & 1.08 & 1.02 & 0.93 & 1.02 \\
\hline 40 & 1.12 & 1.46 & 1.06 & 1.13 & 1.26 & 0.77 & 1.13 \\
\hline 80 & n.a. & 1.36 & n.a. & n.a. & n.a. & 0.88 & 1.12 \\
\hline 160 & n.a. & n.a. & n.a. & n.a. & n.a. & n.a. & n.a. \\
\hline 320 & n.a. & n.a. & n.a. & n.a. & n.a. & n.a. & n.a. \\
\hline Average & 1.04 & 1.30 & 0.95 & 1.08 & 1.14 & 0.88 & 1.08 \\
\hline$r$ & 0.55 & 0.66 & 1.00 & 1.00 & 0.55 & -0.49 & 0.77 \\
\hline $\operatorname{LSD}_{(0.01)}$ & \multicolumn{7}{|c|}{ Cobalt dose -0.09 , kind of amendment -0.09 , interaction -0.22} \\
\hline
\end{tabular}

$r$, correlation coefficient; $n$.a., not analysed

\subsection{Magnesium}

In the objects without amendments, a 3rd cobalt dose ( $80 \mathrm{mg} \mathrm{Co} \mathrm{kg}^{-1}$ of soil) had no significant effect on magnesium content of main crop both organs and on its content of after-crop shoots (Table 6). Only after application of 2 nd cobalt dose ( $40 \mathrm{mg} \mathrm{Co} \mathrm{kg}{ }^{-1}$ of soil) the magnesium content of white mustard roots significantly increased 
Table 3 Potassium content in plants

\begin{tabular}{|c|c|c|c|c|c|c|c|}
\hline \multirow{2}{*}{$\begin{array}{l}\text { Cobalt dose }\left(\mathrm{mg} \mathrm{kg}^{-1}\right. \\
\text { of soil) }\end{array}$} & \multicolumn{7}{|c|}{ Kind of amendment } \\
\hline & $\begin{array}{l}\text { Without } \\
\text { amendments }\end{array}$ & Manure & Clay & Charcoal & Zeolite & $\begin{array}{l}\text { Calcium } \\
\text { oxide }\end{array}$ & Average \\
\hline \multicolumn{8}{|l|}{ Spring barley—shoots } \\
\hline 0 & 16.81 & 16.92 & 15.52 & 17.30 & 16.53 & 16.75 & 16.64 \\
\hline 20 & 17.25 & 16.99 & 16.45 & 18.03 & 15.54 & 17.42 & 16.95 \\
\hline 40 & 17.21 & 16.87 & 17.21 & 17.88 & 15.90 & 17.82 & 17.15 \\
\hline 80 & 17.98 & 17.15 & 17.16 & 18.31 & 16.36 & 17.04 & 17.33 \\
\hline 160 & n.a. & 17.18 & 17.56 & 18.97 & 16.22 & 17.90 & 17.57 \\
\hline 320 & n.a. & 19.08 & n.a. & n.a. & n.a. & 18.03 & 18.56 \\
\hline Average & 17.31 & 17.37 & 16.78 & 18.10 & 16.11 & 17.50 & 17.36 \\
\hline$r$ & 0.96 & 0.93 & 0.81 & 0.95 & 0.18 & 0.68 & 0.99 \\
\hline $\operatorname{LSD}_{(0.01)}$ & \multicolumn{7}{|c|}{ Cobalt dose -0.31 , kind of amendment -0.31 , interaction -0.77} \\
\hline \multicolumn{8}{|l|}{ Spring barley—roots } \\
\hline 0 & 15.09 & 13.61 & 14.98 & 16.22 & 14.26 & 15.61 & 14.96 \\
\hline 20 & 14.86 & 15.18 & 15.48 & 16.33 & 13.50 & 15.39 & 15.12 \\
\hline 40 & 13.56 & 15.42 & 14.47 & 15.71 & 13.60 & 15.56 & 14.72 \\
\hline 80 & 13.90 & 15.06 & 14.32 & 15.27 & 12.12 & 14.62 & 14.22 \\
\hline 160 & n.a. & 14.96 & n.a. & n.a. & n.a. & 13.96 & 14.46 \\
\hline 320 & n.a. & 16.00 & n.a. & n.a. & n.a. & n.a. & 16.00 \\
\hline Average & 14.35 & 15.04 & 14.81 & 15.88 & 13.37 & 15.03 & 14.91 \\
\hline$r$ & -0.76 & 0.66 & -0.74 & -0.93 & -0.96 & -0.96 & 0.57 \\
\hline $\operatorname{LSD}_{(0.01)}$ & \multicolumn{7}{|c|}{ Cobalt dose -0.16 , kind of amendment -0.16 , interaction -0.39} \\
\hline \multicolumn{8}{|l|}{ White mustard—shoots } \\
\hline 0 & 17.34 & 18.17 & 17.85 & 18.29 & 18.03 & 19.32 & 18.17 \\
\hline 20 & 18.36 & 19.56 & 18.50 & 19.15 & 16.44 & 19.82 & 18.64 \\
\hline 40 & 19.90 & 20.42 & 18.79 & 20.39 & 16.84 & 18.96 & 19.21 \\
\hline 80 & 20.29 & 20.43 & 20.00 & 22.41 & 18.17 & 18.96 & 20.04 \\
\hline 160 & n.a. & 19.64 & n.a. & n.a. & n.a. & 21.86 & 20.75 \\
\hline 320 & n.a. & n.a. & n.a. & n.a. & n.a. & n.a. & n.a. \\
\hline Average & 18.97 & 19.64 & 18.78 & 20.06 & 17.37 & 19.79 & 19.36 \\
\hline$r$ & 0.93 & 0.39 & 0.99 & 1.00 & 0.28 & 0.76 & 0.97 \\
\hline $\operatorname{LSD}_{(0.01)}$ & \multicolumn{7}{|c|}{ Cobalt dose -0.01 , kind of amendment -0.01 , interaction -0.03} \\
\hline \multicolumn{8}{|l|}{ White mustard—roots } \\
\hline 0 & 14.13 & 17.17 & 14.31 & 15.19 & 13.33 & 15.21 & 14.89 \\
\hline 20 & 15.02 & 16.34 & 14.79 & 15.96 & 11.06 & 15.62 & 14.80 \\
\hline 40 & 18.05 & 17.61 & 16.71 & 16.08 & 13.50 & 16.05 & 16.34 \\
\hline 80 & n.a. & 17.90 & n.a. & n.a. & n.a. & 15.47 & 16.68 \\
\hline 160 & n.a. & n.a. & n.a. & n.a. & n.a. & n.a. & n.a. \\
\hline 320 & n.a. & n.a. & n.a. & n.a. & n.a. & n.a. & n.a. \\
\hline Average & 15.74 & 17.26 & 15.27 & 15.74 & 12.63 & 15.59 & 15.68 \\
\hline$r$ & 0.95 & 0.68 & 0.95 & 0.92 & 0.06 & 0.26 & 0.90 \\
\hline $\operatorname{LSD}_{(0.01)}$ & \multicolumn{7}{|c|}{ Cobalt dose -0.16 , kind of amendment -0.16 , interaction -0.38} \\
\hline
\end{tabular}

$r$, correlation coefficient; $n$.a., not analysed (by $17 \%, r=0.92$ ). Soil amendments did not affect the content of this macroelement in spring barley both organs or white mustard shoots. Among all amendments only the effect of manure was positive, as it lead to a $9 \%$ increase in magnesium content in after-crop roots, compared to the control block (without amendments). 
Table 4 Sodium content in plants

\begin{tabular}{|c|c|c|c|c|c|c|c|}
\hline \multirow{2}{*}{$\begin{array}{l}\text { Cobalt dose ( } \mathrm{mg} \mathrm{kg}^{-1} \\
\text { of soil) }\end{array}$} & \multicolumn{7}{|c|}{ Kind of amendment } \\
\hline & $\begin{array}{l}\text { Without } \\
\text { amendments }\end{array}$ & Manure & Clay & Charcoal & Zeolite & $\begin{array}{l}\text { Calcium } \\
\text { oxide }\end{array}$ & Average \\
\hline \multicolumn{8}{|l|}{ Spring barley—shoots } \\
\hline 0 & 0.65 & 1.46 & 0.65 & 0.81 & 1.58 & 0.77 & 0.98 \\
\hline 20 & 0.69 & 1.53 & 0.67 & 0.82 & 1.57 & 0.79 & 1.01 \\
\hline 40 & 0.74 & 1.50 & 0.84 & 0.92 & 1.78 & 0.73 & 1.09 \\
\hline 80 & 0.83 & 1.63 & 0.88 & 1.01 & 1.83 & 0.80 & 1.16 \\
\hline 160 & n.a. & 1.66 & 0.80 & 1.67 & 0.79 & 0.86 & 1.15 \\
\hline 320 & n.a. & 0.64 & n.a. & n.a. & n.a. & 0.81 & 0.73 \\
\hline Average & 0.73 & 1.40 & 0.77 & 1.04 & 1.51 & 0.79 & 1.02 \\
\hline$r$ & 1.00 & -0.78 & 0.57 & 0.96 & -0.73 & 0.59 & -0.61 \\
\hline $\operatorname{LSD}_{(0.01)}$ & \multicolumn{7}{|c|}{ Cobalt dose -0.05 , kind of amendment -0.05 , interaction -0.13} \\
\hline \multicolumn{8}{|l|}{ Spring barley—roots } \\
\hline 0 & 0.83 & 0.88 & 0.99 & 0.88 & 1.48 & 0.97 & 1.00 \\
\hline 20 & 0.86 & 1.42 & 1.02 & 0.90 & 1.40 & 0.93 & 1.09 \\
\hline 40 & 0.92 & 1.46 & 1.05 & 0.91 & 1.54 & 0.95 & 1.14 \\
\hline 80 & 1.12 & 1.50 & 1.14 & 1.00 & 1.73 & 0.98 & 1.24 \\
\hline 160 & n.a. & 1.57 & n.a. & n.a. & n.a. & 1.19 & 1.38 \\
\hline 320 & n.a. & 1.82 & n.a. & n.a. & n.a. & n.a. & 1.82 \\
\hline Average & 0.93 & 1.44 & 1.05 & 0.92 & 1.54 & 1.00 & 1.28 \\
\hline$r$ & 0.98 & 0.79 & 0.99 & 0.96 & 0.89 & 0.91 & 1.00 \\
\hline $\operatorname{LSD}_{(0.01)}$ & \multicolumn{7}{|c|}{ Cobalt dose -0.02 , kind of amendment -0.02 , interaction -0.05} \\
\hline \multicolumn{8}{|l|}{ White mustard—shoots } \\
\hline 0 & 1.29 & 1.95 & 1.31 & 1.06 & 2.38 & 1.19 & 1.53 \\
\hline 20 & 1.33 & 2.04 & 1.37 & 1.22 & 2.28 & 1.29 & 1.59 \\
\hline 40 & 1.55 & 2.10 & 1.53 & 1.21 & 2.53 & 1.27 & 1.70 \\
\hline 80 & 1.48 & 2.22 & 1.68 & 1.55 & 2.47 & 1.36 & 1.79 \\
\hline 160 & n.a. & 2.43 & n.a. & n.a. & n.a. & 1.51 & 1.97 \\
\hline 320 & n.a. & n.a. & n.a. & n.a. & n.a. & n.a. & n.a. \\
\hline Average & 1.41 & 2.15 & 1.47 & 1.26 & 2.41 & 1.32 & 1.72 \\
\hline$r$ & 0.73 & 1.00 & 0.98 & 0.96 & 0.56 & 0.98 & 0.99 \\
\hline $\operatorname{LSD}_{(0.01)}$ & \multicolumn{7}{|c|}{ Cobalt dose -0.04 , kind of amendment -0.04 , interaction -0.10} \\
\hline \multicolumn{8}{|l|}{ White mustard—roots } \\
\hline 0 & 1.27 & 1.83 & 1.26 & 1.11 & 1.95 & 1.18 & 1.43 \\
\hline 20 & 1.30 & 1.82 & 1.41 & 1.18 & 1.90 & 1.22 & 1.47 \\
\hline 40 & 1.47 & 1.81 & 1.48 & 1.21 & 2.04 & 1.29 & 1.55 \\
\hline 80 & n.a. & 1.79 & n.a. & n.a. & n.a. & 1.33 & 1.56 \\
\hline 160 & n.a. & n.a. & n.a. & n.a. & n.a. & n.a. & n.a. \\
\hline 320 & n.a. & n.a. & n.a. & n.a. & n.a. & n.a. & n.a. \\
\hline Average & 1.35 & 1.81 & 1.38 & 1.17 & 1.96 & 1.25 & 1.50 \\
\hline$r$ & 0.94 & -1.00 & 0.98 & 0.97 & 0.65 & 0.97 & 0.91 \\
\hline $\operatorname{LSD}_{(0.01)}$ & \multicolumn{7}{|c|}{ Cobalt dose -0.02 , kind of amendment -0.02 , interaction -0.04} \\
\hline
\end{tabular}

$r$, correlation coefficient; $n$.a., not analysed

\subsection{PCA analysis and correlation coefficients}

The conducted PCA analysis (Figs. 1, 2, 3, 4, 5, 6, 7, and 8) and calculated correlation coefficients (Table 7) confirmed the significant relations between contents of particular macroelements in organs of both plant species.

Figure 1 shows the contents of macroelements in spring barley shoots under the influence of cobalt and the selected 
Table 5 Calcium content in plants

\begin{tabular}{|c|c|c|c|c|c|c|c|}
\hline \multirow{2}{*}{$\begin{array}{l}\text { Cobalt dose ( } \mathrm{mg} \mathrm{kg}^{-1} \\
\text { of soil) }\end{array}$} & \multicolumn{7}{|c|}{ Kind of amendment } \\
\hline & $\begin{array}{l}\text { Without } \\
\text { amendments }\end{array}$ & Manure & Clay & Charcoal & Zeolite & $\begin{array}{l}\text { Calcium } \\
\text { oxide }\end{array}$ & Average \\
\hline \multicolumn{8}{|l|}{ Spring barley — shoots } \\
\hline 0 & 0.47 & 0.14 & 0.32 & 0.28 & 0.32 & 0.41 & 0.32 \\
\hline 20 & 0.36 & 0.18 & 0.41 & 0.35 & 0.32 & 0.43 & 0.34 \\
\hline 40 & 0.42 & 0.16 & 0.44 & 0.38 & 0.40 & 0.63 & 0.40 \\
\hline 80 & 0.59 & 0.24 & 0.53 & 0.55 & 0.37 & 0.57 & 0.47 \\
\hline 160 & n.a. & 0.32 & 0.81 & 0.82 & 0.79 & 0.82 & 0.71 \\
\hline 320 & n.a. & 0.38 & n.a. & n.a. & n.a. & 1.18 & 0.78 \\
\hline Average & 0.46 & 0.24 & 0.50 & 0.47 & 0.44 & 0.67 & 0.51 \\
\hline$r$ & 0.67 & 0.95 & 0.99 & 1.00 & 0.93 & 0.98 & 0.95 \\
\hline $\operatorname{LSD}_{(0.01)}$ & \multicolumn{7}{|c|}{ Cobalt dose -0.04 , kind of amendment -0.04 , interaction -0.10} \\
\hline \multicolumn{8}{|l|}{ Spring barley—roots } \\
\hline 0 & 0.15 & 0.44 & 0.22 & 0.23 & 0.19 & 0.26 & 0.25 \\
\hline 20 & 0.15 & 0.23 & 0.20 & 0.23 & 0.20 & 0.28 & 0.22 \\
\hline 40 & 0.21 & 0.16 & 0.25 & 0.24 & 0.23 & 0.29 & 0.23 \\
\hline 80 & 0.35 & 0.22 & 0.34 & 0.39 & 0.26 & 0.32 & 0.31 \\
\hline 160 & n.a. & 0.24 & n.a. & n.a. & n.a. & 0.57 & 0.40 \\
\hline 320 & n.a. & 0.28 & n.a. & n.a. & n.a. & n.a. & 0.28 \\
\hline Average & 0.22 & 0.26 & 0.25 & 0.27 & 0.22 & 0.35 & 0.28 \\
\hline$r$ & 0.96 & -0.10 & 0.93 & 0.92 & 1.00 & 0.95 & 0.43 \\
\hline $\operatorname{LSD}_{(0.01)}$ & \multicolumn{7}{|c|}{ Cobalt dose -0.00 , kind of amendment -0.00 , interaction -0.01} \\
\hline \multicolumn{8}{|l|}{ White mustard-shoots } \\
\hline 0 & 1.01 & 0.84 & 1.03 & 0.96 & 1.07 & 1.12 & 1.00 \\
\hline 20 & 1.14 & 0.91 & 1.18 & 1.07 & 1.03 & 1.17 & 1.08 \\
\hline 40 & 1.32 & 1.00 & 1.31 & 1.14 & 1.16 & 1.21 & 1.19 \\
\hline 80 & 1.50 & 1.08 & 1.43 & 1.33 & 1.30 & 1.33 & 1.33 \\
\hline 160 & n.a. & 1.30 & n.a. & n.a. & n.a. & 1.52 & 1.41 \\
\hline 320 & n.a. & n.a. & n.a. & n.a. & n.a. & n.a. & n.a. \\
\hline Average & 1.24 & 1.03 & 1.24 & 1.13 & 1.14 & 1.27 & 1.21 \\
\hline$r$ & 0.99 & 0.99 & 0.97 & 1.00 & 0.93 & 1.00 & 0.95 \\
\hline $\operatorname{LSD}_{(0.01)}$ & \multicolumn{7}{|c|}{ Cobalt dose -0.04 , kind of amendment -0.04 , interaction -0.09} \\
\hline \multicolumn{8}{|l|}{ White mustard—roots } \\
\hline 0 & 0.17 & 0.19 & 0.19 & 0.24 & 0.27 & 0.24 & 0.22 \\
\hline 20 & 0.20 & 0.21 & 0.26 & 0.24 & 0.21 & 0.24 & 0.23 \\
\hline 40 & 0.36 & 0.24 & 0.29 & 0.19 & 0.33 & 0.43 & 0.31 \\
\hline 80 & n.a. & 0.29 & n.a. & n.a. & n.a. & 0.41 & 0.35 \\
\hline 160 & n.a. & n.a. & n.a. & n.a. & n.a. & n.a. & n.a. \\
\hline 320 & n.a. & n.a. & n.a. & n.a. & n.a. & n.a. & n.a. \\
\hline Average & 0.24 & 0.23 & 0.25 & 0.23 & 0.27 & 0.33 & 0.28 \\
\hline$r$ & 0.94 & 1.00 & 0.96 & -0.85 & 0.50 & 0.80 & 0.96 \\
\hline $\operatorname{LSD}_{(0.01)}$ & \multicolumn{7}{|c|}{ Cobalt dose -0.02 , kind of amendment -0.02 , interaction -0.06} \\
\hline
\end{tabular}

$r$, correlation coefficient; $n$.a., not analysed amendments, using PCA analysis. The contents of calcium, potassium, magnesium, and nitrogen accounted for $51.02 \%$ of total correlation of data set, and the contents of sodium and phosphorus accounted for $26.60 \%$. The potassium vector was shortest of the remaining macroelement vectors, which indicates the smallest effect was in share of variability. In first group of macroelements, the occurrence of a strong positive correlation was noted between nitrogen and magnesium, while 
Table 6 Magnesium content in plants

\begin{tabular}{|c|c|c|c|c|c|c|c|}
\hline \multirow{2}{*}{$\begin{array}{l}\text { Cobalt dose }\left(\mathrm{mg} \mathrm{kg}^{-1}\right. \\
\text { of soil) }\end{array}$} & \multicolumn{7}{|c|}{ Kind of amendment } \\
\hline & $\begin{array}{l}\text { Without } \\
\text { amendments }\end{array}$ & Manure & Clay & Charcoal & Zeolite & $\begin{array}{l}\text { Calcium } \\
\text { oxide }\end{array}$ & Average \\
\hline \multicolumn{8}{|l|}{ Spring barley—shoots } \\
\hline 0 & 3.13 & 3.10 & 3.11 & 3.10 & 3.13 & 3.11 & 3.11 \\
\hline 20 & 3.13 & 3.11 & 3.13 & 3.14 & 3.14 & 3.12 & 3.13 \\
\hline 40 & 3.15 & 3.11 & 3.15 & 3.13 & 3.14 & 3.15 & 3.14 \\
\hline 80 & 3.17 & 3.17 & 3.15 & 3.16 & 3.14 & 3.14 & 3.16 \\
\hline 160 & n.a. & 3.20 & 3.22 & 3.24 & 3.23 & 3.17 & 3.21 \\
\hline 320 & n.a. & 3.24 & n.a. & n.a. & n.a. & 3.23 & 3.23 \\
\hline Average & 3.14 & 3.15 & 3.15 & 3.15 & 3.16 & 3.15 & 3.16 \\
\hline$r$ & 0.97 & 0.94 & 0.98 & 0.98 & 0.94 & 0.96 & 0.96 \\
\hline $\operatorname{LSD}_{(0.01)}$ & \multicolumn{7}{|c|}{ Cobalt dose -0.01 , kind of amendment -0.01 , interaction -0.04} \\
\hline \multicolumn{8}{|l|}{ Spring barley—roots } \\
\hline 0 & 2.97 & 3.08 & 2.97 & 2.97 & 2.88 & 2.91 & 2.96 \\
\hline 20 & 3.00 & 3.06 & 2.89 & 2.92 & 2.87 & 2.91 & 2.94 \\
\hline 40 & 3.02 & 3.02 & 3.00 & 2.93 & 2.90 & 2.94 & 2.97 \\
\hline 80 & 3.13 & 3.02 & 3.10 & 3.10 & 3.02 & 2.99 & 3.06 \\
\hline 160 & n.a. & 3.08 & n.a. & n.a. & n.a. & 3.19 & 3.13 \\
\hline 320 & n.a. & 3.13 & n.a. & n.a. & n.a. & n.a. & 3.13 \\
\hline Average & 3.03 & 3.06 & 2.99 & 2.98 & 2.92 & 2.99 & 3.03 \\
\hline$r$ & 0.97 & 0.71 & 0.82 & 0.77 & 0.93 & 0.97 & 0.86 \\
\hline $\operatorname{LSD}_{(0.01)}$ & \multicolumn{7}{|c|}{ Cobalt dose -0.01 , kind of amendment -0.01 , interaction -0.02} \\
\hline \multicolumn{8}{|l|}{ White mustard—shoots } \\
\hline 0 & 3.28 & 3.28 & 3.14 & 3.21 & 3.23 & 3.20 & 3.22 \\
\hline 20 & 3.32 & 3.32 & 3.18 & 3.24 & 3.23 & 3.24 & 3.25 \\
\hline 40 & 3.34 & 3.31 & 3.19 & 3.25 & 3.26 & 3.26 & 3.27 \\
\hline 80 & 3.38 & 3.33 & 3.26 & 3.31 & 3.29 & 3.28 & 3.31 \\
\hline 160 & n.a. & 3.37 & n.a. & n.a. & n.a. & 3.32 & 3.34 \\
\hline 320 & n.a. & n.a. & n.a. & n.a. & n.a. & n.a. & n.a. \\
\hline Average & 3.33 & 3.32 & 3.19 & 3.25 & 3.25 & 3.26 & 3.28 \\
\hline$r$ & 0.99 & 0.96 & 0.98 & 0.99 & 0.98 & 0.95 & 0.98 \\
\hline $\operatorname{LSD}_{(0.01)}$ & \multicolumn{7}{|c|}{ Cobalt dose -0.02 , kind of amendment -0.02 , interaction -0.04} \\
\hline \multicolumn{8}{|l|}{ White mustard—roots } \\
\hline 0 & 2.45 & 2.78 & 2.44 & 2.60 & 2.81 & 2.53 & 2.60 \\
\hline 20 & 2.51 & 2.75 & 2.70 & 2.64 & 2.56 & 2.41 & 2.59 \\
\hline 40 & 2.87 & 2.86 & 2.81 & 2.49 & 2.76 & 2.79 & 2.76 \\
\hline 80 & n.a. & 3.00 & n.a. & n.a. & n.a. & 2.76 & 2.88 \\
\hline 160 & n.a. & n.a. & n.a. & n.a. & n.a. & n.a. & n.a. \\
\hline 320 & n.a. & n.a. & n.a. & n.a. & n.a. & n.a. & n.a. \\
\hline Average & 2.61 & 2.85 & 2.65 & 2.57 & 2.71 & 2.62 & 2.71 \\
\hline$r$ & 0.92 & 0.95 & 0.97 & -0.73 & -0.20 & 0.72 & 0.95 \\
\hline $\operatorname{LSD}_{(0.01)}$ & \multicolumn{7}{|c|}{ Cobalt dose -0.08, kind of amendment -0.08 , interaction -0.19} \\
\hline
\end{tabular}

$r$, correlation coefficient; $n$.a., not analysed in second group of macroelements, there was a strong positive correlation between sodium and phosphorus. A weaker positive correlation was also noted between calcium and potassium. Figure 2 indicates that zeolite and manure had greatest effect (and charcoal and calcium oxide had smallest effect) on contents of analysed macroelements in spring barley shoots.

The contents of the macroelements concerned in spring barley roots, being under influence of both test factors used 
Table 7 Correlation coefficients $(r)$ between macroelement content in plants

\begin{tabular}{|c|c|c|c|c|c|}
\hline & $\mathrm{P}$ & $\mathrm{K}$ & $\mathrm{Na}$ & $\mathrm{Ca}$ & $\mathrm{Mg}$ \\
\hline \multicolumn{6}{|c|}{ Spring barley—shoots } \\
\hline $\mathrm{N}$ & -0.083 & $0.536 * *$ & -0.010 & $0.636^{* *}$ & $0.894 * *$ \\
\hline $\mathrm{P}$ & & -0.325 & $0.651 * *$ & $-0.374 * *$ & -0.163 \\
\hline $\mathrm{K}$ & & & -0.246 & $0.388^{*}$ & $0.509 * *$ \\
\hline $\mathrm{Na}$ & & & & -0.330 & -0.013 \\
\hline $\mathrm{Ca}$ & & & & & $0.699 * *$ \\
\hline \multicolumn{6}{|c|}{ Spring barley—roots } \\
\hline $\mathrm{N}$ & 0.238 & -0.135 & 0.271 & $0.532 * *$ & $0.569 * *$ \\
\hline $\mathrm{P}$ & & 0.150 & $0.650 * *$ & -0.165 & 0.341 \\
\hline $\mathrm{K}$ & & & -0.302 & -0.237 & -0.167 \\
\hline $\mathrm{Na}$ & & & & -0.096 & 0.197 \\
\hline $\mathrm{Ca}$ & & & & & $0.632 * *$ \\
\hline \multicolumn{6}{|c|}{ White mustard—shoots } \\
\hline $\mathrm{N}$ & $0.734 * *$ & $0.596 * *$ & 0.328 & $0.679 * *$ & $0.616^{* *}$ \\
\hline $\mathrm{P}$ & & 0.180 & $0.611 * *$ & 0.257 & $0.618 * *$ \\
\hline $\mathrm{K}$ & & & -0.207 & $0.497 * *$ & $0.461 * *$ \\
\hline $\mathrm{Na}$ & & & & -0.089 & 0.335 \\
\hline $\mathrm{Ca}$ & & & & & 0.339 \\
\hline \multicolumn{6}{|c|}{ White mustard—roots } \\
\hline $\mathrm{N}$ & $0.452 * *$ & $0.465^{* *}$ & 0.345 & 0.127 & $0.527 * *$ \\
\hline $\mathrm{P}$ & & 0.363 & $0.650 * *$ & -0.186 & $0.544 * *$ \\
\hline $\mathrm{K}$ & & & -0.126 & 0.227 & $0.501 * *$ \\
\hline $\mathrm{Na}$ & & & & 0.030 & $0.589 * *$ \\
\hline $\mathrm{Ca}$ & & & & & $0.574 * *$ \\
\hline
\end{tabular}

Significant for $* * P \leq 0.01, * P \leq 0.05$

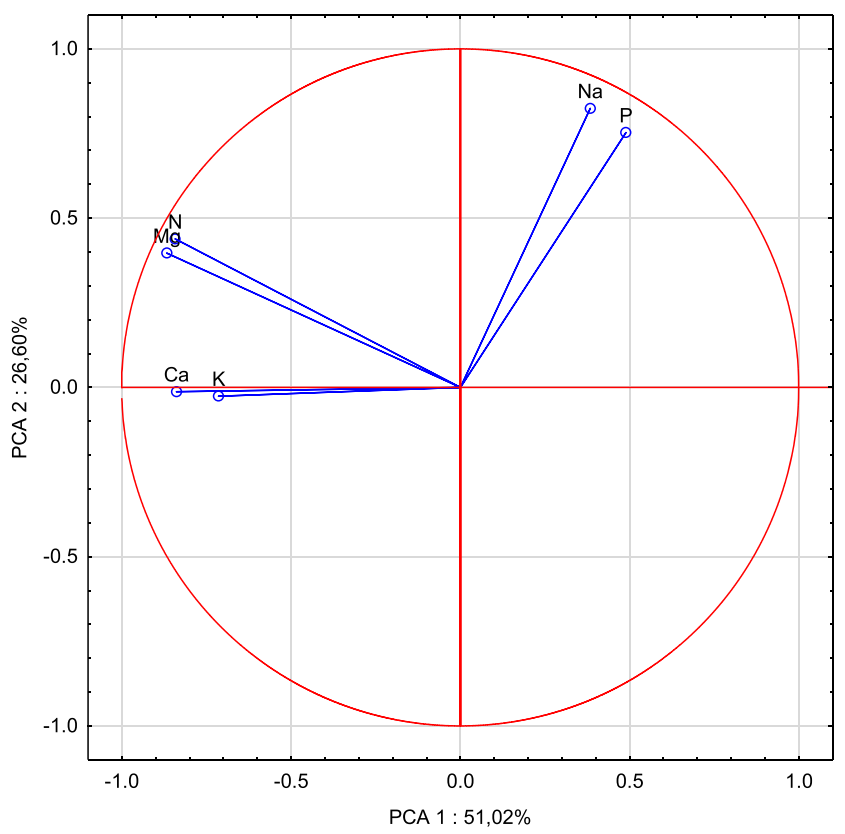

Fig. 1 Macroelement content in spring barley shoots calculated with PCA method. Vectors represent variable $(\mathrm{N}, \mathrm{P}, \mathrm{K}, \mathrm{Na}, \mathrm{Ca}, \mathrm{Mg})$ as PCA results in experiment, are presented in Fig. 3. The first group of microelements, including nitrogen, magnesium, and potassium, accounted for $40.09 \%$ of total correlation of data set, while the second group (phosphorus, sodium, and calcium) accounted for $27.33 \%$. Similarly to shoots of spring barley, the potassium vector was shortest, which indicates its slight effect in the share of variability. A strong positive correlation was noted between nitrogen and magnesium, and between sodium and phosphorus. A strong negative correlation was observed between calcium and potassium. Of the applied soil amendments, manure had maximum effect, and calcium oxide had smallest effect, on the contents of selected macroelements in spring barley roots (Fig. 4).

The PCA analysis presented in Fig. 5 illustrates the effects of cobalt and soil amendments on the macroelement content of white mustard shoots. The total correlation of data set for first group of macroelements (nitrogen, magnesium, and phosphorus) amounted to $52.36 \%$, while for second group (sodium, potassium, and calcium) it amounted to $26.00 \%$. Due to the shortest lengths of magnesium and calcium vectors as compared with other vectors, their effect in share of variability was small. Both in the first and in the second group of macroelements, the occurrence of a strong positive correlation was noted between macroelements - a weaker correlation between nitrogen and magnesium, and a strong correlation between potassium and calcium. No negative correlations between analysed macroelements were found. Manure definitely had maximum impact on the contents of macroelements in the white mustard shoots (Fig. 6).

The effects of cobalt and soil amendments on the contents of macroelements in white mustard roots are shown in Fig. 7. For nitrogen, magnesium, potassium, and phosphorus, the total correlation of data set was $47.45 \%$, while for calcium and sodium, it was $23.39 \%$. Potassium and nitrogen were characterised by shortest lengths of vectors, which explains their slight effects in share of variability. A strong positive correlation was found between phosphorus and sodium and a weaker correlation was found between magnesium and nitrogen. Similarly to white mustard shoots, no negative correlations were noted. Figure 8 indicates that calcium oxide, manure, and charcoal had a greatest effect on macroelement contents of white mustard roots than remaining amendments.

\section{Discussion}

\subsection{Effect of cobalt contamination on macroelement content in plants}

Cobalt belongs to the group of microelements whose content in relation to the dry matter of plants ranges from 0.5 to $10 \mathrm{mg}$ $\mathrm{kg}^{-1}$ (Lohry 2007). At low contents, cobalt stimulates physiological processes, while at high contents it can result in the 
Fig. 2 Effect of amendments and cobalt dose on macroelement content in spring barley shoots calculated with PCA method. Points show element content in plant shoots (WA - without amendments, $\mathrm{M}$ - manure, $\mathrm{L}-$ clay, C-charcoal, Z-zeolite, $\mathrm{CO}$ - calcium oxide, $1-0 \mathrm{mg}$, 2-20 mg, 3-40 mg, 4-80 mg, 5 - $160 \mathrm{mg}, 6$ - $320 \mathrm{mg} \mathrm{Co} \mathrm{kg}^{-1}$ of soil)

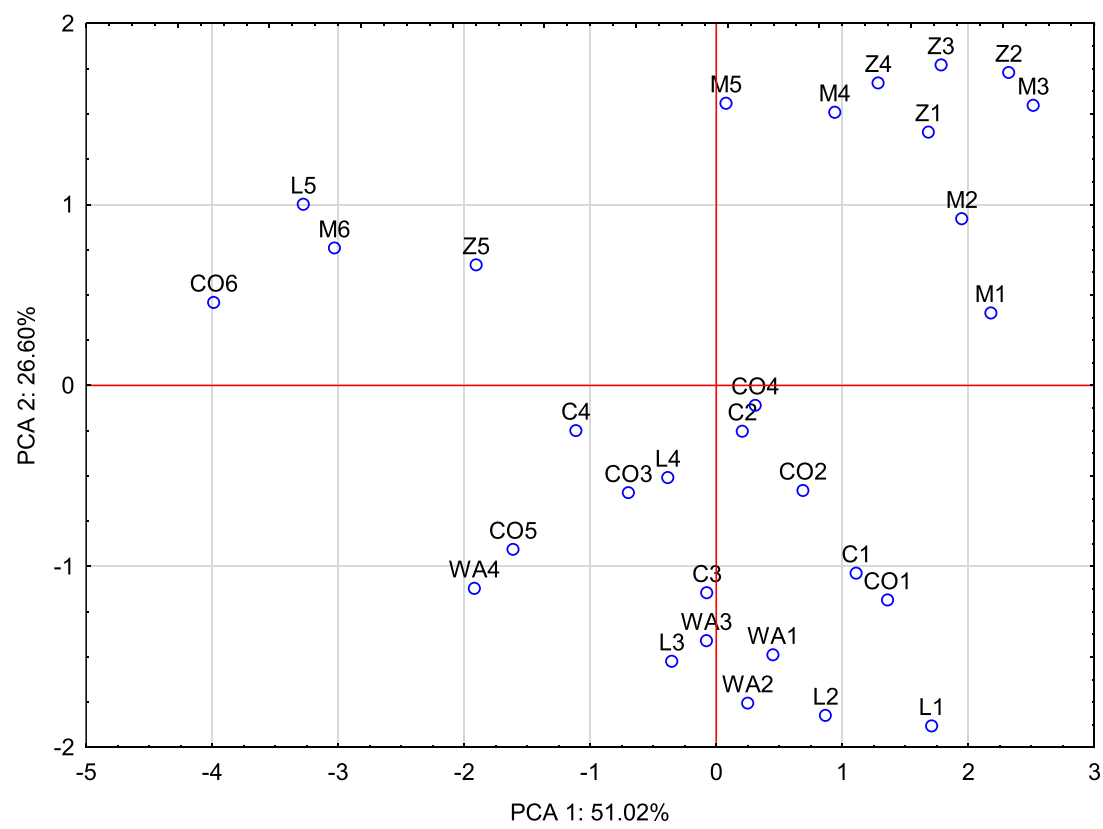

emergence of toxic symptoms, consequently leading to reduced yielding of plants (Ali et al. 2010). The effect of high doses of cobalt on the reduction in yielding of plants was also confirmed in the author's own study. Since for objects with doses of 160 and $320 \mathrm{mg} \mathrm{Co} \mathrm{kg}^{-1}$ of soil (spring barley all organs and white mustard shoots) and with doses of 80, 160, and $320 \mathrm{mg} \mathrm{Co} \mathrm{kg}^{-1}$ of soil (white mustard roots) the gain of plants was slight, it was not possible to perform an analysis of the contents of the macroelements concerned in these objects. Macroelements (phosphorus, nitrogen, potassium, calcium, magnesium, and sulphur) play a very important role in the first

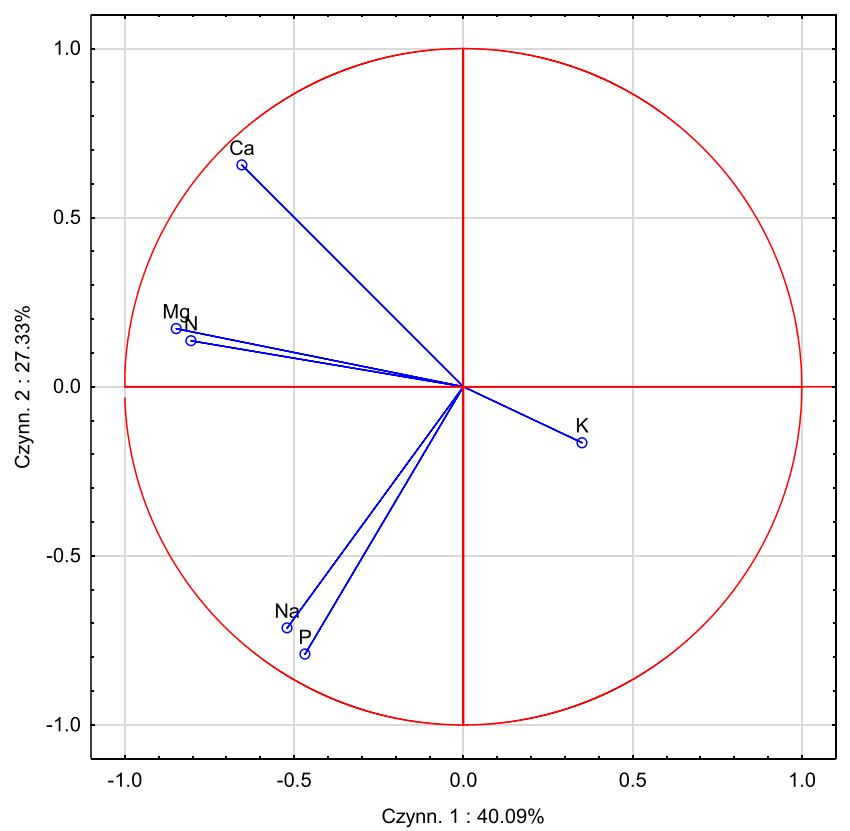

Fig. 3 Macroelement content in spring barley roots calculated with PCA method. Vectors represent variable (N, P, K, Na, Ca, Mg) as PCA results stages of plant vegetation. Macronutrients determine the rapid growth, formation of seeds and fruits, and the correct chemical composition of plants. The correct proportions and the correct macronutrient content of plants are desirable. An imbalance in the level of macronutrient concentrations can affect plant growth, delay fruit ripening, and production of nutrient-poor plants (Al-Obaidi 2020). Kosiorek and Wyszkowski (2016) found that the introduction of increasing doses of cobalt to soil contributed to a decrease in the $\mathrm{pH}$ value. With the decrease in $\mathrm{pH}$ value of the soil, the availability of cobalt increases considerably, which promotes its increased accumulation in plant tissues (Gál et al. 2008). Cobalt contamination of soil increases its content in plant organs, especially in roots (Boros-Lajszner et al. 2020). At high cobalt contents, disturbances to the uptake of other elements occur in a plant, among other symptoms (Chatterjee and Chatterjee 2000; International Plant Nutrition Institute 2015). The above relationship is also confirmed by own study, in particular in the objects with 2nd and $3 \mathrm{rd}$ doses ( 40 and $80 \mathrm{mg} \mathrm{Co} \mathrm{kg}^{-1}$ ), which had maximum impact on an increase in contents of analysed macroelements in both test plants (except phosphorus, whose accumulation decreased in spring barley shoots and in white mustard roots).

As reported by Kabata-Pendias and Pendias (2001), cobalt is necessary in structure of porphyrins, which are responsible for formation of coenzyme cobalamin which is involved in binding $\mathrm{N}_{2}$. Studies by International Plant Nutrition Institute (2015) reveal that fertilising soil with cobalt has a positive effect on the increase of nitrogen, phosphorus, and potassium contents in peanuts. An increase in calcium and magnesium contents and a decrease in sodium content in the stems and roots of tomato (varieties Moneymaker and Edcawy) after the application of $15 \mathrm{mg}$ of cobalt per $\mathrm{kg}$ of soil were 
Fig. 4 Effect of amendments and cobalt dose on macroelement content in spring barley roots calculated with PCA method. Points show element content in plant roots (WA-without amendments, $\mathrm{M}-$ manure, $\mathrm{L}-$ clay, C-charcoal, Z-zeolite, $\mathrm{CO}$ - calcium oxide, $1-0 \mathrm{mg}$, 2-20 mg, 3-40 mg, 4-80 mg, $5-160 \mathrm{mg}, 6$ - $320 \mathrm{mg} \mathrm{Co} \mathrm{kg}{ }^{-1}$ of soil)

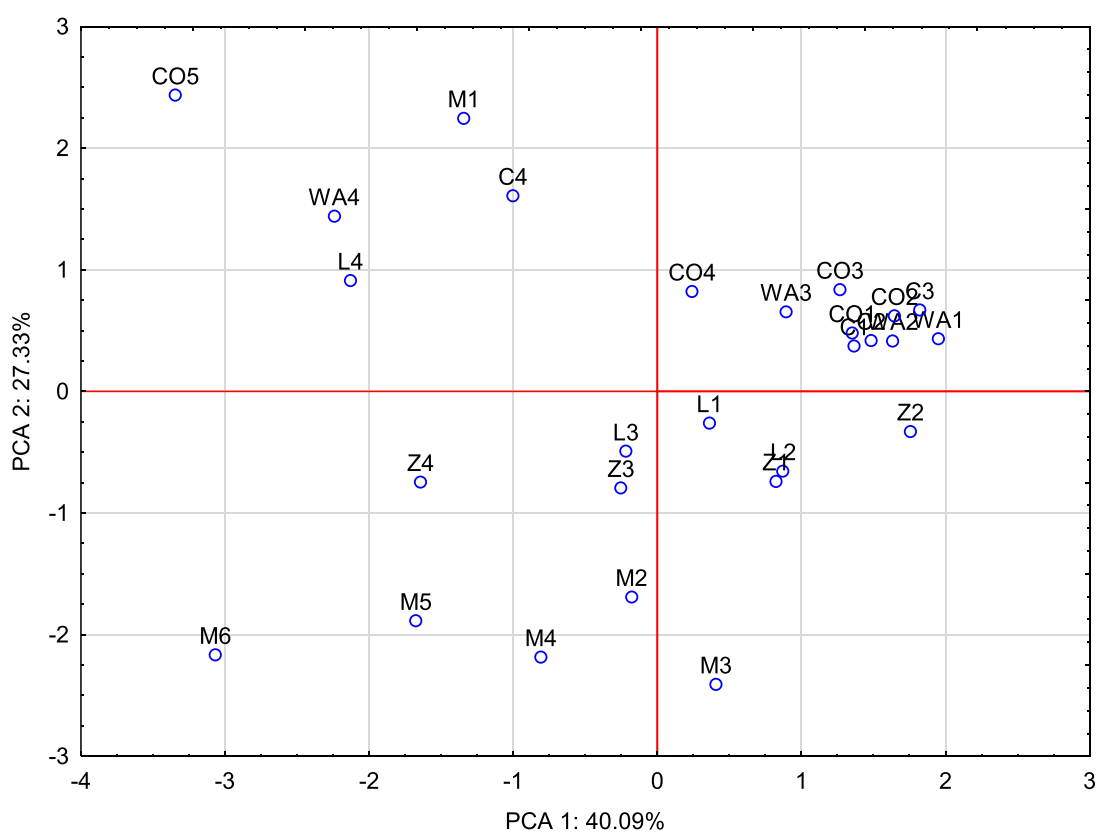

demonstrated by Gad (2005). Increasing doses of cobalt increased the content of majority macroelements (phosphorus, sodium, magnesium, and calcium) and decreased content of potassium in oats (Wyszkowski et al. 2009). In other research, cobalt soil contamination increased the content of nitrogen, phosphorus, calcium, and sodium in oat organs - grain, straw, and roots (Kosiorek and Wyszkowski 2019). The same relations between cobalt contamination and macroelement contents (except potassium) in plants, especially white mustard, were observed in the present study. According to Chatterjee

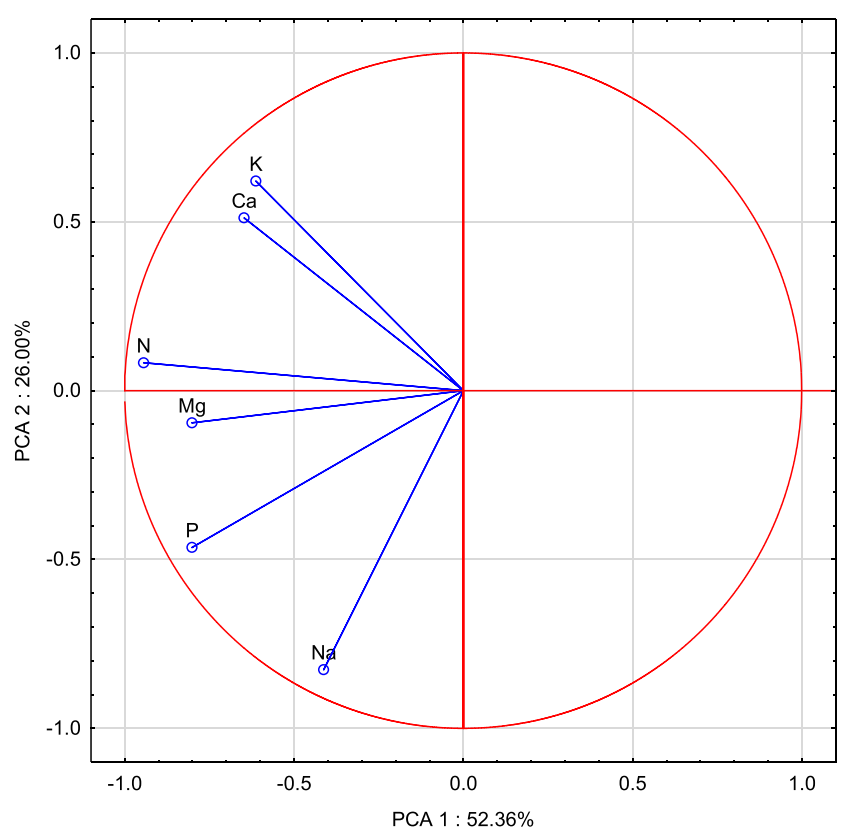

Fig. 5 Macroelement content in white mustard shoots calculated with PCA method. Vectors represent variable $(\mathrm{N}, \mathrm{P}, \mathrm{K}, \mathrm{Na}, \mathrm{Ca}, \mathrm{Mg})$ as PCA results and Chatterjee (2000), a 0.5-mM concentration of cobalt sulphate applied to seedlings of cauliflower Brassica oleracea L., variety Maghi, after 86 days of growth has an effect on the decrease in phosphorus accumulation from roots to the stem, and then to the leaves, which amounts to, respectively, 0.62 $\pm 0.05,0.29 \pm 0.02$, and $0.30 \pm 0.02 \%$.

\subsection{Effect of remediation with amendments on macroelement content in plants}

The negative effect of cobalt and other trace elements on plants can be limited by the application of various amendments, such as organic substance (Liu et al. 2018), liming (Doskočil and Pekař 2012), and other sorbents (Kosiorek and Wyszkowski 2019, 2020). It is especially important to restore the balance in soils, including the correct air-water relations, the number of microorganisms, and soil enzymatic activity. They determine the uptake of nutrients and the correct content and proportions of the elements in plants (BorosLajszner et al. 2020). This has a major impact on the growth and development of plants.

According to Chaudhari et al. (2017), manure is characterised by long-term properties of metal adsorption and has a positive effect on increase of soil quality in terms of biology and on an increase in the content of primary elements in the soil. Palm et al. (2001) concluded that after the introduction of organic fertilisers to the soil, the availability of primary macroelements which have an effect on the growth and development of plants, inter alia nitrogen, phosphorus, and potassium, considerably increases. Compost can lead to an increase in nitrogen content of spring rape and oats (Wyszkowski and Ziółkowska 2009), potassium and calcium 
Fig. 6 Effect of amendments and cobalt dose on macroelement content in white mustard shoots calculated with PCA method. Points show element content in plant shoots (WA - without amendments, $\mathrm{M}$ - manure, $\mathrm{L}-$ clay, C-charcoal, Z-zeolite, $\mathrm{CO}$ - calcium oxide, $1-0 \mathrm{mg}$, 2-20 mg, 3-40 mg, 4-80 mg, $5-160 \mathrm{mg}, 6$ - $320 \mathrm{mg} \mathrm{Co} \mathrm{kg}{ }^{-1}$ of soil)

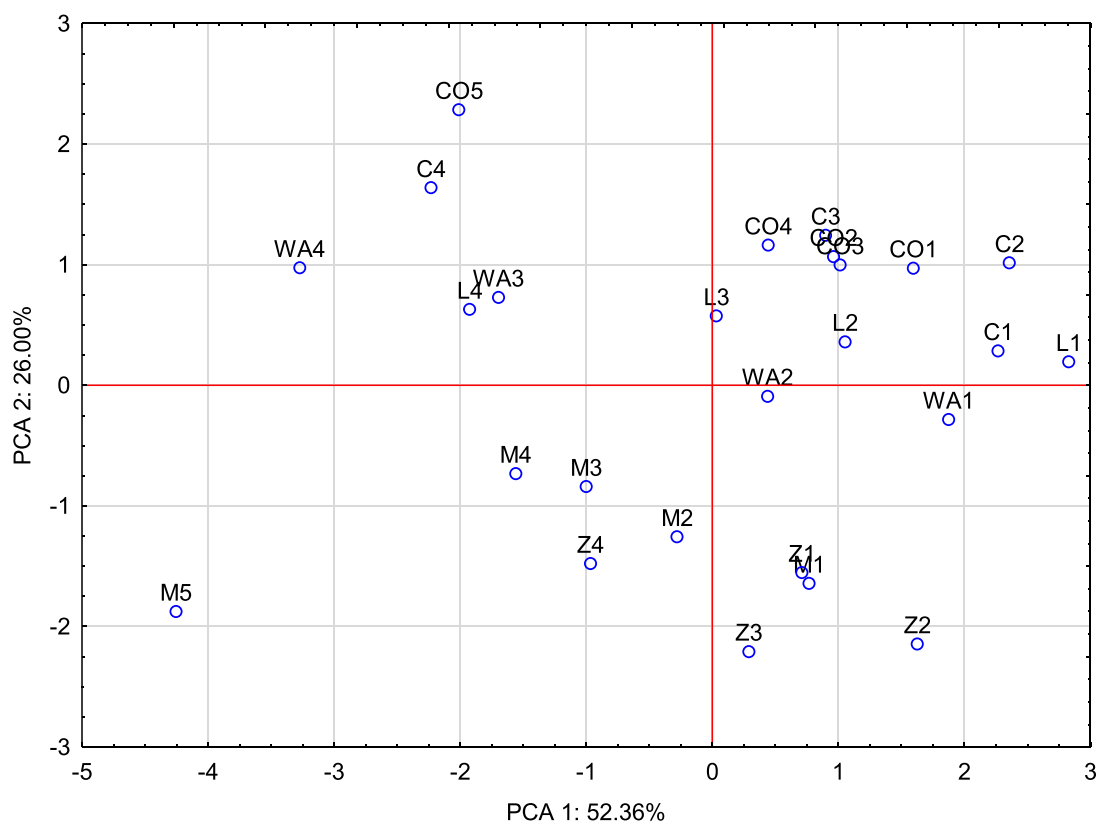

contents of maize shoots (Sivitskaya and Wyszkowski 2013), and the magnesium content of various plant species (Ciećko et al. 2004, 2005). Compost also resulted in a decrease in phosphorus content of maize shoots (Sivitskaya and Wyszkowski 2013) and in potassium content of various plants (Ciećko et al. 2004, 2005). The manure increased the content of nitrogen, phosphorus, potassium, and sodium in all organs of oats - grain, straw, and roots (Kosiorek and Wyszkowski 2019). The above relationships are also confirmed by own study, as in the objects with the addition of manure, an increase in phosphorus content was noted in white mustard and

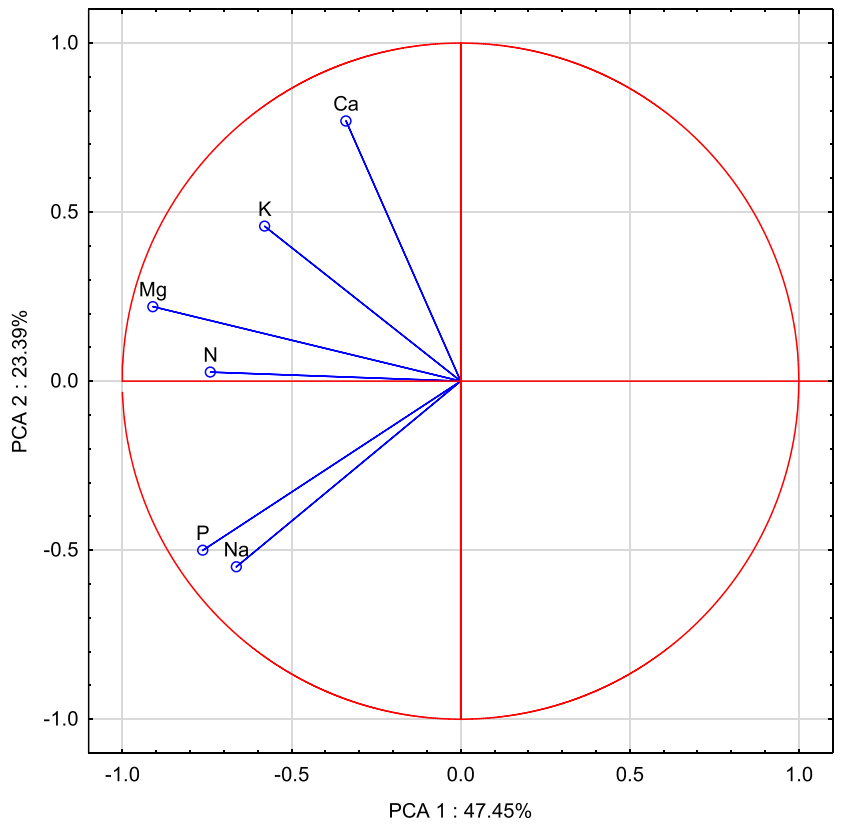

Fig. 7 Macroelement content in white mustard roots calculated with PCA method. Vectors represent variable (N, P, K, Na, Ca, Mg) as PCA results spring barley shoots, and an increase in potassium and magnesium contents in white mustard roots. Mucheru-Muna et al. (2014) found a strong effect of organic fertilisation combined with mineral fertilisation on an increase in contents of the element forms available to plants in the soil.

Kosiorek and Wyszkowski (2016) indicated that application of manure, particularly calcium oxide to soil results in an increase in soil $\mathrm{pH}$ value of, respectively, 6 and approx. 7.5. According to McCauley et al. (2017), maintaining soil $\mathrm{pH}$

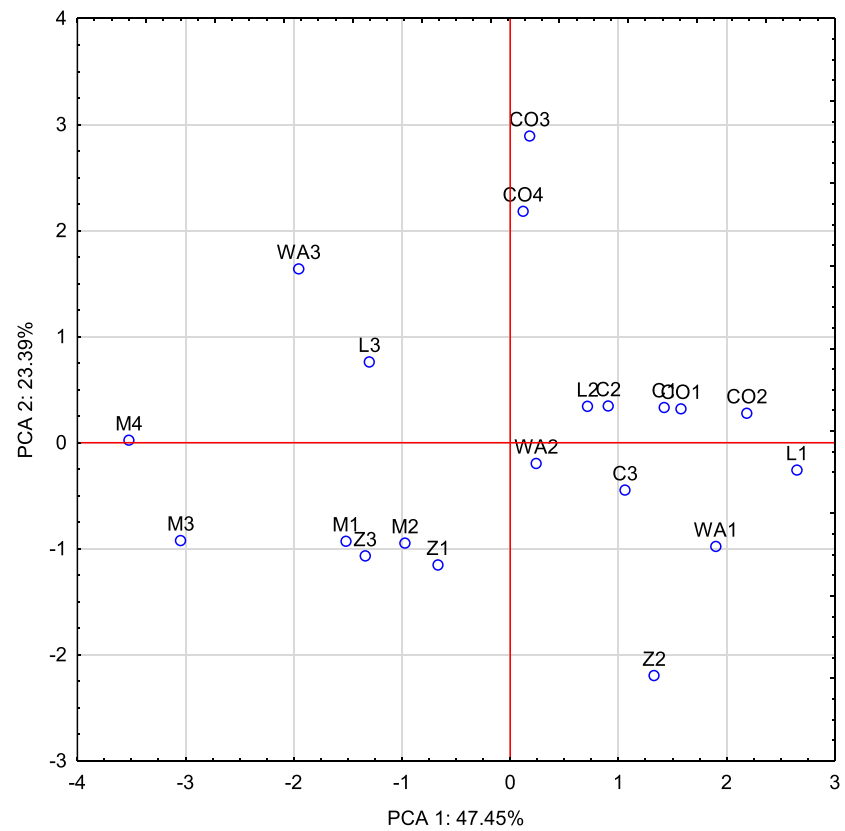

Fig. 8 Effect of amendments and cobalt dose on macroelement content in white mustard roots calculated with PCA method. Points show element content in plant roots (WA-without amendments, $\mathrm{M}-$ manure, $\mathrm{L}-$ clay, C-charcoal, $\mathrm{Z}$ - zeolite, $\mathrm{CO}$ - calcium oxide, $1-0 \mathrm{mg}, 2-20$ $\mathrm{mg}, 3-40 \mathrm{mg}, 4-80 \mathrm{mg}, 5-160 \mathrm{mg}, 6-320 \mathrm{mg} \mathrm{Co} \mathrm{kg}^{-1}$ of soil) 
value at a level from 5.5 to 7.5 increased content of available phosphorus in soil, while from 6.5 to 8 has a positive effect on calcium content. Therefore, it can be assumed that in own study in objects with these amendments, the contents of both macroelements in main and after-crop were the highest. This relationship was confirmed for phosphorus in blocks with manure in white mustard and spring barley roots and for calcium in blocks with calcium oxide in both test plants. In objects with calcium oxide, a much higher soil $\mathrm{pH}$ value was maintained than for manure, which promoted an increase in phosphorus content of soil according to the assumptions of McCauley et al. (2017); however, in this study, an opposite effect was obtained, as the phosphorus content of white mustard decreased. Kaniuczak et al. (2004a, b) demonstrated that liming of soils promotes a decrease in cobalt content of the spring barley grains and of potato tubers and green matter of fodder sunflower. Calcium oxide decreased the content of most macronutrients in oat grain, straw, and roots (Kosiorek and Wyszkowski 2019). Therefore, it can be concluded that manure and calcium oxide applied in this experiment are amendments which not only have a positive effect on properties of soil and an increase in selected macroelement content in test plants, but, most importantly, can lead to reduction toxic cobalt content in plants.

According to Adeyemi and Idowu (2017), the charcoal application to soil considerably increases organic carbon content of soil, which translates into an improvement in soil fertility. Kolb et al. (2008) found that presence of carbon amendments in four moderate soils, namely Mollisol, Alfisol, Entisol, and Spodosol, significantly increases both bacterial and microbial activity in soil and availability of elements. A positive effect of charcoal on increase in availability of selected macroelements was also noted in own study, as in objects with addition of charcoal, there was a significant increase in nitrogen content of spring barley shoots, and in the potassium content of spring barley all organs and white mustard shoots. The effect of charcoal and zeolite on the macroelement content in oat organs was less equivocal (Kosiorek and Wyszkowski 2019).

As reported by Mumpton (1999) and Kithome et al. (1999), zeolites are amendments that exhibit potent properties of both precipitation and water accumulation and the ability to exchange cations with no significant changes appearing in their structure. In turn, the application of zeolites in agriculture has a major influence on the increase in phosphorus and potassium contents in a soil poor in elements (Dwairi 1998). Zeolite can increase the nitrogen content of spring rape and oats (Wyszkowski and Ziółkowska 2009), phosphorus, magnesium, and calcium contents of various plant species (Sivitskaya and Wyszkowski 2013; Wyszkowski and Modrzewska 2015), as well as potassium content, and a decrease in phosphorus content of maize shoots (Sivitskaya and Wyszkowski 2013). In own study, zeolite only increased phosphorus content of the main crop shoots and sodium content of both plants, while for potassium, it exhibited an opposite effect. The negative effect of zeolite on potassium content in plants was observed in Manolov et al. (2005) experiment.

In the research by Kosiorek and Wyszkowski (2020), spring barley and white mustard were plants suitable for phytoremediation, especially phytostabilisation, as they retained cobalt well in the roots.

\section{Conclusions}

Cobalt-contaminated soil and amendments application to soil significantly differentiated macroelement content of all organs of test plants. In objects without amendments, a 3rd cobalt dose ( $80 \mathrm{mg} \mathrm{Co} \mathrm{kg}^{-1}$ of soil) contributed the most to an increase in nitrogen, sodium, and calcium contents of both organs of spring barley, and an increase in the potassium content of its shoots. Phosphorus content increased in roots and decreased in shoots of this plant. Cobalt application to soil resulted in a decrease in potassium content of spring barley roots. Under influence of cobalt, the contents of all macroelements in white mustard organs increased. Application of amendments to soil had the strong impact on differentiation of phosphorus, sodium, and calcium contents of all organs of both plant species, compared to the control block (without amendments). Among the amendments, the clearest and most potent impact had manure, which increased the phosphorus, potassium, and sodium contents of all organs of both plants. It should also be stressed that zeolite addition to soil caused a large increase in sodium content, and calcium oxide application increased calcium content of both plants. The effects of clay and charcoal on the macroelement contents of plants were less clear.

Funding The project was financed by the University of Warmia and Mazury in Olsztyn (Poland) as part of the topic No. 20.610.003-110.

Open Access This article is licensed under a Creative Commons Attribution 4.0 International License, which permits use, sharing, adaptation, distribution and reproduction in any medium or format, as long as you give appropriate credit to the original author(s) and the source, provide a link to the Creative Commons licence, and indicate if changes were made. The images or other third party material in this article are included in the article's Creative Commons licence, unless indicated otherwise in a credit line to the material. If material is not included in the article's Creative Commons licence and your intended use is not permitted by statutory regulation or exceeds the permitted use, you will need to obtain permission directly from the copyright holder. To view a copy of this licence, visit http://creativecommons.org/licenses/by/4.0/. 


\section{References}

Adeyemi TOA, Idowu OD (2017) Biochar: Promoting crop yield, improving soil fertility, mitigating climate change and restoring polluted soils. World News Nat Sci 8:27-36

Ali B, Hayat S, Hayat Q, Ahmad A (2010) Cobalt stress affects nitrogen metabolism, photosynthesis and antioxidant system in chickpea (Cicer arietinum L.). J Plant Interact 5(3):223-231

Al-Obaidi JR (2020) Micro- and macronutrient signalling in plant cells: a proteomic standpoint under stress conditions. In: Aftab T, Hakeem KR (eds) Plant Micronutrients. Springer, Cham

ATSDR (2004) Toxicological profile for cobalt. US Departament of Health and Human Services Agency for Toxic Substances and Diseases Registry: 1-397

Awa SH, Hadibarata T (2020) Removal of heavy metals in contaminated soil by phytoremediation mechanism: a review. Water Air Soil Pollut 231(47):1-15

Baker AJM, Reeves RD, Hajar ASM (1994) Heavy metal accumulation and tolerance in British populations of the metallophyte Thlaspi caerulescems J.E\&C-Presl (Brassicaceae). New Phytol 127(1):6168. https://doi.org/10.1111/j.1469-8137.1994.tb04259.x

Boros-Lajszner E, Wyszkowska J, Kucharski J (2020) Phytoremediation of soil contaminated with nickel, cadmium and cobalt. Int $\mathrm{J}$ Phytorem 23:252-262. https://doi.org/10.1080/15226514.2020. 1807907

Bremner JM (1965) Total nitrogen. In: Methods of soil analysis, part 2. Chemical and microbiological properties. Black CA et al. (eds). American Society of Agronomy, Madison. Agronomy 9:1149-1178

Brodowska MS, Kaczor A (2009) The effect of various forms of sulphur and nitrogen on calcium and magnesium content and uptake in spring wheat (Triticum aestivum L.) and cocksfoot (Dactylis glomerata L.). J Elem 14(4):641-647

Chatterjee J, Chatterjee C (2000) Phytotoxicity of cobalt, chromium and copper in cauliflower. Environ Pollut 109(1):69-74

Chaudhari BH, Parmar JK, Mali RH, Bumbadiya NH (2017) Effect of Co level and FYM on growth and yield of fodder maize. Int $\mathrm{J}$ Chem Stud 5(1):618-622

Ciećko Z, Kalembasa S, Wyszkowski M, Rolka E (2004) Effect of soil contamination by cadmium on potassium uptake by plants. Pol Environ St 13(3):333-337

Ciećko Z, Kalembasa S, Wyszkowski M, Rolka E (2005) The magnesium content in plants on soil contaminated with cadmium. Pol J Environ St 14(3):365-370

Collins RN, Kinsela AS (2011) Pedogenic factors and measurements of the plant uptake of cobalt. Plant Soil 339:499-512

Dell Inc (2016) Dell Statistica (data analysis software system), version 13. software.dell.com

Doskočil L, Pekař M (2012) Removal of metal ions from a multicomponent mixture using natural lignite. Fuel Process Technol 101:29-34

Dwairi IM (1998) Evaluation of Jordanian zeolite tuff as a controlled slow-release fertilizer for $\mathrm{NH}_{4}{ }^{+}$. Environ Geol 34:1-4

Gad N (2005) Interactive effect of cobalt and salinity on tomato plants Igrowth and mineral composition as affected by cobalt and salinity. Res J Agric Biol Sci 1(3):261-269

Gál J, Hursthouse A, Tatner P, Stewart F, Welton R (2008) Cobalt and secondary poisoning in the terrestrial food chain: data review and research gaps to support risk assessment. Environ Int 34(6):821-838

Gąsiorowska B, Makarewicz A, Nowosielska A (2011) The content of selected macroelements in maize cultivars grain sowed in three dates. Fragm Agron 28(3):7-15

Grzesik M, Romanowska-Duda Z (2009) New technologies of the energy plant production in the predicted climate changed conditions. Bjuleten Djerżawnowo Nikitskowo Botaniczieskowo Sada. Ukrainska Akademia Agrarnych Nauk 99:65-68
International Plant Nutrition Institute (2015) Cobalt. Nutri-facts. Agronomic fact sheets on crop nutrients. North American Edition $15: 1-2$

Kabata-Pendias A, Pendias H (2001) Trace elements in soils and plants, 3rd edn. CRC Press, Boca Raton, pp 1-413

Kaniuczak J, Hajduk E, Rożek D (2004a) Effect of liming and mineral fertilization on cobalt content in plants cultivated in crop rotation. Part I. Cobalt content in potatoes and fodder sunflower. Adv Agr Sci Probl Issue 502:109-115

Kaniuczak J, Hajduk E, Rożek D (2004b) Effect of liming and mineral fertilization on cobalt content in plants cultivated in crop rotation. Part II. Cobalt content in grains of winter wheat and spring barley. Adv Agr Sci Probl Issue 502:117-123

Kithome M, Paul JW, Lavkulich LM, Bomke AA (1999) Effect of pH on ammonium adsorption by natural zeolite clinoptilolite. Commun Soil Sci Plant Anal 30(9-10):1417-1430

Kolb SE, Fermanich KJ, Dornbush ME (2008) Effect of charcoal quantity on microbial biomass and activity in temperate soils. Soil Biol Biochem 73(4):1173-1181

Kosiorek M, Wyszkowski W (2016) Effect of neutralising substances on selected properties of soil contaminated with cobalt. J Ecol Eng 17(3):193-197

Kosiorek M, Wyszkowski W (2019) Content of macronutrients in oat (Avena sativa L.) after remediation of soil polluted with cobalt. Environ Monit Assess 191(389):1-15

Kosiorek M, Wyszkowski M (2020) Remediation of cobalt-contaminated soil using manure, clay, charcoal, zeolite, calcium oxide, main crop (Hordeum vulgare L.), and after-crop (Synapis alba L.). Minerals 10(5):429

Kwiatkowska J, Maciejewska A (2008) Influence of organic matter forms on physico-chemical properties of soil and content of organic carbon. Soil Sci Annu 59(1):128-133

Lange B, van der Ent A, Baker AJM, Echevarria G, Mahy G, Malaisse F, Meerts P, Pourret O, Verbruggen N, Faucon M-P (2017) Copper and cobalt accumulation in plants: a critical assessment of the current state of knowledge. New Phytol 213(2):537-551

Liu L, Li W, Song W, Guo M (2018) Remediation techniques for heavy metal-contaminated soils: principles and applicability. Sci Total Environ 633:206-219

Lohry R (2007) Micronutrients: functions, sources and application methods. Indiana CCA Conference Proceedings:1-15

Loomis WE, Shull CA (1937) Methods in plant physiology. McGraw Hill, New York and London, pp 1-472

Manolov ID, Antonov G, Stoilov I, Baev T, Baev M (2005) Jordanian zeolitic tuff as a raw material for the preparation of substrates used for plant growth. J Cent Eur Agric 6(4):485-494

McCauley A, Jones C, Olson-Rutz K (2017) Soil pH and organic matter. Nutr Manage Mod 8:1-16

Morrissey J, Baxter IR, Lee J, Li L, Lahner B, Grotz N, Kaplan J, Salt DE, Guerinot ML (2009) The ferroportin metal efflux proteins function in iron and cobalt homeostasis in Arabidopsis. Plant Cell 21:33263338

Mucheru-Muna MW, Mugendi D, Pypers P, Mugwe J, Kung'u J, Vanlauwe B, Merckx R (2014) Enhancing maize productivity and profitability using organic inputs and mineral fertilizer in central Kenya small-hold fields. Exp Agric 50(2):250-269

Mumpton FA (1999) Uses of natural zeolites in agriculture and industry. Proc Natl Acad Sci U S A 96(7):3463-3470

Ostrowska A, Gawliński S, Szczubiałka Z (1991) Methods for analysis and evaluation of soil and plant properties. Institute of Environmental Protection - National Research Institute, Warsaw: $1-334$

Palm CA, Gachengo CN, Delve RJ, Cadisch G, Giller KE (2001) Organic inputs for soil fertility management in tropical agroecosystems: application of an organic resource database. Agric Ecosyst Environ 83: $27-42$ 
Pszczółkowski W, Romanowska-Duda Z, Pszczółkowska A, Grzesik M, Wysokińska Z (2012) Application of phytoremediation in restoring sustainable development to the environment: economic and soil conditions. Comp Econ Res Centr East Eur 15(3):37-55

Regulation (2016) Regulation of Minister of the Environment of 1 September 2016 on the procedures for the assessment of land surface contamination. J Laws:1395

Ronan E (2007) Micro-elements in agriculture. Pract Hydropon Greenhous: $39-48$

Saad L, Parmentier I, Colinet G, Malaisse F, Faucon MP, Meerts P, Mahy $G$ (2012) Investigating the vegetation-soil relationships on the copper-cobalt rock outcrops of Katanga (D. R. Congo), an essential step in a biodiversity conservation plan. Restor Ecol 20(3):405-415

Saleh HM, Aglan RF, Mahmoud HH (2019) Ludwigia stolonifera for remediation of toxic metals from simulated wastewater. Chem Ecol 35(2):164-178

Sharma CP (2006) Plant micronutrients. Science Publishers, EnfieldJersey-Plymouth: 1-265

Sivitskaya V, Wyszkowski M (2013) Changes in the content of some macroelements in maize (Zea mays L.) under effect of fuel oil after application of different substances to soil. J Elem 18(4):705-714

Stanisławska-Glubiak E, Korzeniowska J (2007) Principles of fertilization with microelements of arable crops. Stud Rep IUNG-PIB 8:99110 (In Polish) van den Brink S, Kleijn R, Sprecher B, Tukker A (2020) Identifying supply risks by mapping the cobalt supply chain. Resour Conserv Recycl 156:104743. https://doi.org/10.1016/j.resconrec.2020. 104743

Wyszkowski M, Modrzewska B (2015) Effect of neutralizing substances on zinc-contaminated soil on the yield and macronutrient content of yellow lupine (Lupinus luteus L.). J Elem 20(2):503-512

Wyszkowski M, Ziółkowska A (2009) Role of compost, bentonite and calcium oxide in restricting the effect of soil contamination with petrol and diesel oil on plants. Chemosphere 74(6):860-865

Wyszkowski M, Wyszkowska J, Radziemska M (2009) Macroelement content in yield of oats (Avena sativa L.) cultivated on soils contaminated with copper, zinc, tin, cobalt and manganese. Ecol Chem Eng A 16(10):1387-1394

Zaborowska M, Kucharski J, Wyszkowska J (2016) Biological activity of soil contaminated with cobalt, tin and molybdenum. Environ Monit Assess 188(7):398

Publisher's note Springer Nature remains neutral with regard to jurisdictional claims in published maps and institutional affiliations. 